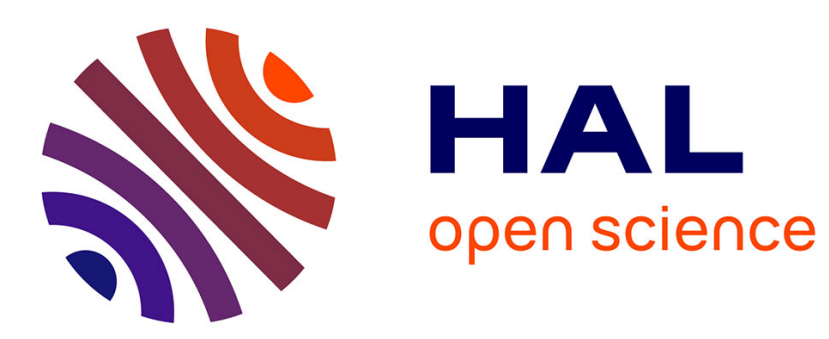

\title{
Le trauma et l'émotion: apports de la théorie janetienne et perspectives nouvelles
}

\author{
Hervé Landa, Guy Gimenez
}

\section{To cite this version:}

Hervé Landa, Guy Gimenez. Le trauma et l'émotion : apports de la théorie janetienne et perspectives nouvelles . L'Évolution Psychiatrique, 2009, 74 (4), 10.1016/j.evopsy.2009.09.006 . hal-01389342

\section{HAL Id: hal-01389342 \\ https://hal-amu.archives-ouvertes.fr/hal-01389342}

Submitted on 28 Oct 2016

HAL is a multi-disciplinary open access archive for the deposit and dissemination of scientific research documents, whether they are published or not. The documents may come from teaching and research institutions in France or abroad, or from public or private research centers.
L'archive ouverte pluridisciplinaire HAL, est destinée au dépôt et à la diffusion de documents scientifiques de niveau recherche, publiés ou non, émanant des établissements d'enseignement et de recherche français ou étrangers, des laboratoires publics ou privés. 


\title{
Trauma
}

\section{Le trauma et l'émotion: apports de la théorie janetienne et perspectives nouvelles}

\author{
Trauma and emotion: contributions of the \\ janetian theory and new perspectives \\ Hervé Landa ${ }^{\mathrm{a}, *}$, Guy Gimenez ${ }^{\mathrm{b}}$
}

\begin{abstract}
a Psychologue clinicien, doctorant, centre PsyCLE (psychologie de la connaissance, du langage et de l'émotion), UFK de psychologie, université de Provence, 29, avenue Robert-Schuman, 13621 Aix-en-Provence cedex 1, France

${ }^{\mathrm{b}}$ Maître de conférences, centre PsyCLE (psychologie de la connaissance, du langage et de l'émotion), UFR de psychologie, université de Provence, 29, avenue Robert-Schuman, 13621 Aix-en-Provence cedex 1, France
\end{abstract}

\section{Résumé}

Les conceptions actuelles du trauma associent une symptomatologie au vécu d'un événement possédant des caractéristiques précises. La symptomatologie se caractérise par un syndrome de répétition considéré comme pathognomonique. Il consiste en une reviviscence de l'expérience traumatique sous la forme de flash-backs, cauchemars, vécus «as if » se manifestant de manière intrusive dans le psychisme du sujet. Les recherches actuelles ont montré qu'il existait dans l'expérience du sujet un vécu dissociatif constitué d'une dépersonnalisation ou d'une déréalisation dont la présence au moment du trauma pouvait être prédictive de la gravité du trauma. Cette dissociation péri-traumatique est rapprochée de la dissociation telle que l'a définie Janet et qui consiste en une désagrégation psychique. Celle-ci comporte une réduction du champ de la conscience et une difficulté de synthèse due à un bouleversement émotionnel. Actuellement, les travaux de Frijda (1985) [1] autour de la fonction des émotions et de leur considération en termes de motivation peuvent nous aider à donner à la dissociation un sens spécifique. Celle-ci ne serait alors plus liée à une désagrégation, mais se rapprocherait d'un évitement psychique radical. Cet évitement serait le propre d'une émotion spécifique, l'effroi, dont la tendance serait une motivation dissociative. Cette hypothèse repose sur la distinction du trauma psychique d'un autre trauma tel qu'il apparaîtrait dans l'hystérie et que Janet considérait sans distinction. Le trauma aurait alors pour conséquence de fixer les images et les sensations associées au vécu. La répétition se manifesterait par la réapparition de cette fixation lorsque l'état de conscience de

q Toute référence à cet article doit porter mention : Landa H, Gimenez G. Le trauma et l'émotion: apports de la théorie janetienne et perspectives nouvelles. Evol psychiatr 2009; 74 .

Auteur correspondant.

Adresse e-mail : hervelanda@gmail.com (H. Landa). 
l'individu se modifie. Nous voyons comment la notion de dissociation chez Janet nous permet, par sa complexité, de considérer sous un jour nouveau la question du trauma et ses manifestations. Elle implique que soit étudiées en toute rigueur les manifestations psychopathologiques au-delà des a priori qui nous amènent trop souvent à créer un mécanisme ad hoc pour expliquer un phénomène. La référence aux travaux de Janet dans le cadre des recherches actuelles ouvre de nouvelles perspectives de recherche autour d'une définition du trauma, de la place de la dissociation péri-traumatique et de la dissociation post-traumatique, des émotions et de la répétition.

\author{
Mots clés : Traumatisme psychique ; Dissociation ; Émotion ; Effroi ; Répétition
}

\begin{abstract}
The current designs of trauma associate a symptomatology with specific characteristics of an event. Symptomatology is characterized by a syndrome of repetition regarded as pathognomonic. It consists of a reviviscence of the traumatic experiment in the form of flashback, nightmares, lived "as if" in an intrusive way in psychism of the subject. Current research showed that there exist a dissociative experience made up of depersonalization or derealisation whose, presence at the time of the experience, could predict the gravity of the trauma. This peri-traumatic dissociation is brought closer to dissociation such as defined Janet and which consists of a psychic disintegration. This one comprises a reduction of the field of the conscience and a difficulty of synthesis due to an emotional upheaval. Currently, work of Frijda (1985) around the function of the emotions and their consideration in terms of motivation can help us to give to dissociation a specific direction. This one would not be then related any more to disintegration, but would approach a radical psychic avoidance. This avoidance would be the characteristic of a specific emotion, the fright, whose tendency would be a dissociative motivation. This hypothesis rests on the distinction of the psychic trauma of another trauma such as it appears in hysteria and that Janet considered without distinction. The trauma would result then in to fix the images and the feelings associated with experience. The repetition would appear by the reappearance of this fixing when the state of consciousness of the individual changes. We see how the concept of dissociation at Janet enables us, by his complexity, to consider in a new light the question of the trauma and its demonstrations. It implies that the psychopathological demonstrations are studied in any rigor beyond the a priori which too often lead us to create an ad hoc mechanism to explain a phenomenon. The reference to work of Janet within the framework of current research opens new prospects of research around a definition of the trauma, place of peri-traumatic dissociation and post-traumatic dissociation, emotions and repetition.
\end{abstract}

Keywords: Trauma; Dissociation; Emotions; Fear; Repetition

La théorie janetienne connaît aujourd'hui un renouveau qu'elle doit en partie aux recherches sur le traumatisme et ses conséquences sur la vie psychique. Ces recherches mettent l'accent sur un certain nombre de concepts qui, après l'apport de la psychanalyse, prennent une dimension nouvelle. Il en est ainsi de la notion de dissociation qui naîtra de l'observation de l'anesthésie hystérique [2] et du questionnement autour de l'unité du moi [3]. D'autres notions naîtront des recherches de Janet qui proposera une théorie du trauma, du bouleversement émotionnel et du symptôme.

Nous voudrions, dans cet article, revenir sur un certain nombre d'aspects de cette théorie et les rapprocher de conceptions modernes du trauma et de l'émotion. Nous évoquerons ainsi les travaux de Nico Frijda (2003) [4] sur l'émotion qui, reprenant la tradition philosophique, considère les émotions comme des passions. De même, nous verrons comment la notion de dissociation est venue correspondre à un certain type de trauma considéré comme 
une expérience violente venant mettre en danger la vie ou l'intégrité physique ou psychique de l'individu.

Nous proposerons alors, à la lumière de l'exposé, une possible interprétation du phénomène dissociatif en termes émotionnels.

\section{Le traumatisme}

Examiner la question du trauma chez Janet demande, tout d'abord, que soit respectée sa prudence vis-à-vis de la généralisation. En effet, rien n'indique, pour lui, que la symptomatologie psychiatrique puisse être examinée, à tout coup, sous l'angle d'un événement venant perturber l'équilibre psychique. Le trauma n'est en ce sens pas étiologique, il constitue davantage un déclencheur de la maladie. Janet (1889) [2] insiste sur la prédisposition de l'individu à être bouleversé par les émotions et à voir son champ de conscience se désagréger. Dans ce cadre, la séduction sexuelle ne représente qu'une des formes de trauma possible.

S'il existe de nombreuses explications cherchant à rendre compte de l'étiologie du traumatisme, il apparaît aujourd'hui un certain consensus en ce qui concerne sa description symptomatologique. Les auteurs s'accordent en effet, et la clinique en rend compte, sur la présence d'un syndrome dit pathognomonique, de répétition ou de reviviscence [5]. Outre cet aspect, il existe un certain nombre d'éléments essentiels à la description du traumatisme et à son diagnostic. Nous nous appuierons pour sa description principalement sur le Diagnostic and Statistical Manual of Mental disorders (DSM-IV) [6] et les travaux de L. Crocq [5].

Il faut tout d'abord évoquer la présence, dans l'histoire du patient, d'un événement possédant des caractéristiques propres comme sa soudaineté, sa violence, la menace qu'il représente pour l'intégrité physique de la personne. Si cet événement possède un certain nombre d'éléments objectifs qui en font un événement potentiellement traumatique, il reste que celui-ci doit être vécu, expériencé sur un mode traumatique. Entre ainsi en compte l'aspect subjectif du trauma qui veut que toute personne confrontée au même événement n'est pas forcément traumatisée. Certains auteurs [7] parleront pour exprimer ce double aspect de «rencontre» postulant ainsi qu'il existe chez le sujet un potentiel traumatique lié à sa nature même. L'expérience traumatique exprimerait ainsi au sujet une part de lui-même qu'il ignorait jusque-là.

L. Crocq (1999) [5] propose une description de l'événement traumatique en termes de facteurs. Il recense ainsi certaines spécificités traumatiques d'un événement. Ces facteurs peuvent être :

- la menace de mort;

- la blessure;

- la souffrance;

- la torture;

- le spectacle de la mort d'autrui.

D'autres auteurs [8] mettront l'accent sur l'expérience de la mort imminente qu'un tel événement peut créer. Il apparaît, en effet, que cette expérience soit à l'origine d'une confrontation au néant dont la caractéristique est d'être impensable. Par là, l'image traumatique ne pourrait se lier au réseau de représentations constitutif du psychisme. Cette image vécue comme un corps étranger interne aurait, dans une certaine mesure, un destin propre.

Comme nous le voyons, le trauma posséderait donc un double aspect, celui d'un événement réel en lien avec une perception et celui d'un vécu en lien avec l'idée de la mort. 


\section{Répétition et fixation}

Les patients que nous rencontrons présentent, ceci permet d'ailleurs de poser le diagnostic, un ensemble de réactions se caractérisant par leur aspect répétitif. Ces réactions ont toutes un aspect intrusif et débordant venant empêcher les patients de reprendre une vie normale. Ceux-ci évoquent, en effet, les ruminations permanentes qui envahissent leur esprit, les cauchemars qui présentent sans cesse la scène traumatique, les flash-backs intervenant à n'importe quel moment de la journée, les sensations éprouvées au moment du trauma. Toutes ces manifestations indiquent l'existence d'un syndrome de répétition qui signe de manière pathognomonique la présence du trauma.

Ces manifestations possèdent un caractère si vivace et réel que $\mathrm{L}$. Crocq préfère parler de reviviscence. Les sujets paraissent, en effet, littéralement revivre la scène traumatique au point de perdre momentanément contact avec la réalité. Ils semblent ainsi, non pas se rappeler, mais bel et bien se transporter à nouveau dans la situation traumatique elle-même. Outre cette répétition qui se manifeste par une apparition et une disparition des troubles, il apparait que certains sujets maintiennent des attitudes, des conduites apparues au moment de l'expérience traumatique. Ainsi a-t-on des témoignages relatant que tel soldat gardait son bras paralysé dans l'action qu'il tentait d'accomplir au moment où il s'est trouvé confronté à la mort de son compagnon. Il s'agit alors d'une véritable fixation, c'est-à-dire du maintien et de la permanence d'une attitude apparue au moment de l'expérience traumatique.

Ce type de fixation est régulièrement étudié dans le cadre d'un phénomène aussi observable que sont les conduites. Pourtant, nous pouvons supposer que la fixation consiste en un phénomène plus général touchant le vécu psychique, les images et les sensations apparues lors du trauma. En parlant de reviviscence, L. Crocq (1999) [5] souligne que c'est l'être dans son entier qui se trouve remis en situation traumatique. En ce sens, il ne s'agit pas du retour d'un souvenir, mais bel et bien d'une fixation du sujet à la situation. Nous pourrions dire qu'une part du sujet s'est maintenue dans la situation irrésolue du trauma. Ceci nous amène à considérer un autre aspect souvent négligé par les auteurs français, il s'agit de la dissociation.

\section{La dissociation}

Ce qu'il est tout d'abord important de noter, c'est que la «Spaltung» utilisée par la suite par Bleuler en référence à la schizophrénie est avant tout pour Freud et Janet référée à l'étude de l'hystérie. Freud créera en 1893 le concept de refoulement mettant ainsi fin à cette confusion, du moins dans sa théorie de la sexualité. Il utilisera pourtant avant cette période les concepts de «Spaltung » et de «Dissoziation» pour expliquer l'éjection d'une représentation hors de la conscience. Mais Freud préfère insister sur l'importance de l'aspect affectif et sur les états hypnoïdes plutôt que de reprendre la notion de désagrégation (propre à Janet).

Nous ne reprendrons pas ici l'étude étymologique du terme qui renvoie «à un mouvement de séparation, de rupture, de désagrégation, de disjonction » [9], mais aussi de scission (en référence à la schizophrénie de Bleuler [10] pour nous intéresser davantage aux aspects psychologiques).

Toutefois, notons que Thoret retient trois modes de significations du mot «dissociation »: [9].

- un sens «social» qui s'attache au groupe,

- un sens «mécanique » qui touche la jonction d'un ensemble d'éléments, 
- et un sens « chimique » qui souligne la séparation de composées qui soit restent autonomes, soit se regroupent par affinité.

Aujourd'hui, de nombreux travaux américains tentent de définir la dissociation en la plaçant sur un continuum allant, par exemple, d'un sentiment de dépersonnalisation à une double identité [11], ce que la classification de l'American Psychiatric Association (APA) semble promulguer puisque le trouble «Personnalité multiple» est devenu «Trouble dissociatif de l'identité». C'est dans le cadre du traumatisme psychique, ou état de stress post-traumatique, que la dissociation est étudiée d'une manière spécifique.

«La dissociation apparaît être le mécanisme par lequel les sensations intenses et les expériences émotionnelles sont déconnectées du domaine social, du langage et de la mémoire, le mécanisme interne par lequel les personnes terrorisées sont réduites au silence (silenced)» (Herman Jl. [12] dans Shilony E, Fleming MZ, 1994 [13]). Pour Gershuny et Thayer (1999) [14], elle « implique une sorte d'accès divisé ou parallèle à un état de conscience dans lequel deux ou plusieurs processus ou contenus mentaux ne sont pas associés ou intégrés et la conscience que les émotions et les pensées sont diminuées ou évitées. La dissociation doit être comprise comme un état altéré ou fragmenté de conscience dans lequel l'expérience est compartimentée » Outre l'aspect de fragmentation, apparaît ici l'idée d'une mise sous silence, d'une compartimentation venant empêcher l'intégration de l'information.

La dissociation est considérée, dans ces études, comme un mécanisme qui apparaît lorsque la souffrance émotionnelle est telle qu'elle n'est plus supportable. Elle devient alors le seul moyen pour la personne de fuir, émotionnellement et cognitivement, ceci en altérant la conscience. Elle apparaît donc lorsque la fuite ou l'attaque ne sont plus possibles.

L'utilisation d'un tel mécanisme aurait un «coût » psychique important dont l'état de détresse post-traumatique serait le résultat. En effet, lorsque l'état dissociatif se maintient, les processus informatif et émotionnel ne peuvent prendre en charge l'expérience traumatique, ce qui conduit à l'apparition d'un état de stress post-traumatique.

Nous voyons que la dissociation est ici, d'une part, traitée en termes d'altération du fonctionnement psychique ce qui nous rapproche de la désagrégation telle que Janet la décrit. Les processus mentaux, et plus particulièrement la capacité de synthèse, n'auraient plus la possibilité de rapporter l'expérience vécue à la conscience unifiée. Ceci aurait pour effet d'isoler un certain nombre d'éléments psychiques, et plus particulièrement des sensations pour Janet. soumis alors à l'automatisme mental. D'autre part, il apparaît que la dissociation puisse aussi être considérée comme un mécanisme psychique permettant la fuite face à un bouleversement psychoaffectif. Cet aspect lui donne un sens intentionnel qui n'était pas présent chez Janet. Il permet d'étendre la dissociation à une tentative d'évitement considérée alors comme une défense présente chez le sujet et se déclenchant dans des circonstances spécifiques. Par là, la dissociation se rapproche d'un mécanisme autonome et général comme celui de refoulement. Il reste à voir en quoi consiste ce mode spécifique d'évitement et quelles en sont les bases psychologiques.

La dissociation, ici péri-traumatique, aurait un nombre de manifestations dont les patients font état. Il s'agit, en effet, de dépersonnalisation, d'une «expérience prolongée ou récurrente d'un sentiment de détachement et d'une impression d'être devenu un observateur extérieur de son propre fonctionnement mental ou de son propre corps (p. ex. sentiment d'être dans un rêve) » (DSM-IV, 1996) [6] de déréalisation, le sentiment bizarre de ne plus reconnaître le monde comme familier, ni même réel, d'amnésie dissociative. 


\section{La dissociation selon Pierre Janet}

Pour P. Janet, la dissociation est avant tout liée à la «désagrégation psychologique ». Il décrit ce phénomène dans sa thèse de doctorat en philosophie sur l' «automatisme psychologique » où, dans un chapitre consacré à la suggestion, il explique qu'il existe chez l'être humain un champ de conscience (comme il existe un champ visuel) dont une de ses particularités est de pouvoir être rétréci.

«Le rétrécissement du champ de la conscience amène à sa suite une grave conséquence, c'est que tous les phénomènes psychologiques ne sont plus synthétisés dans une même perception personnelle et qu'un certain nombre d'entre eux restent isolés et non perçus. Cette remarque importante nous a conduit à l'étude des phénomènes subconscients et de la division de la personnalité » ([2], p. 15).

Pour expliquer l'effet de ce rétrécissement, il faut concevoir que la fonction de la conscience est de synthétiser les images en représentations. Cette capacité de synthèse varierait d'une personne à une autre, mais constituerait la marque d'un état de conscience. Par ce biais, Janet maintient qu'il existe une unité du psychisme, introduit la notion de subconscient et soutient qu'il existe une hiérarchie du fonctionnement psychique, la capacité de synthèse étant la plus haute de ces fonctions. La complexité des actes accomplis par les personnes en état de somnambulisme, comme l'écriture automatique, laissent en effet penser qu'il existe bien une seconde forme de pensée. L'automatisme qui régit cette pensée ne peut alors être confondu avec un simple mécanisme psychologique. Nous voyons que dans cette conception, la notion de dissociation est liée à une dégradation de la capacité de synthèse.

Pour illustrer ses propos, Janet étudie l'automatisme total puis l'automatisme partiel. La catalepsie et le somnambulisme montrent qu'il existe chez les personnes dans un de ces deux états une perte sensorielle. Cette perte consiste en une incapacité à utiliser les images correspondant à l'ensemble des sens, dont la kinesthésie à laquelle l'auteur donne une très grande importance.

«Quand un sens ou même une sensibilité plus spéciale a disparu, les images et par conséquent les souvenirs des phénomènes qui ont été autrefois fournis par ce sens ont disparu également» ([2], p. 144). Janet constate ainsi que Lucie, une de ses patientes, ne peut retrouver de souvenirs d'enfance visuels dans certains états. D'après lui, les patientes hystériques n'ont pas perdu la sensation, ni même l'image, mais ne peuvent plus associer ces images au reste des représentations présentes en mémoire.

«Le somnambulisme change ces images prédominantes, sans donner précisément des sensibilités nouvelles; il relève de leur effacement certaines images particulières et en fait un centre nouveau autour duquel la pensée s'oriente d'une manière différente » ([2], p. 125).

«Une forme particulière d'oubli résulte donc de la distraction, comme une autre résultait de l'amnésie » ([2], p. 125).

L'oubli des rêves au réveil serait ainsi dû, non pas à une perte mnésique, mais à un changement des représentations associées liées à l'état de conscience.

La désagrégation aurait pour effet d'isoler une partie du psychisme qui répondrait aux lois de l'automatisme et dont les éléments, pouvant parfois se regrouper, donneraient naissance à un nouveau «système, une personnalité indépendante de la première » ([2], p. 418). Janet étudie plusieurs cas d'hystérie et montre que certaines personnes tombent dans un état somnambulique naturel où les souvenirs correspondent à ceux de l'état précédent. Pour distinguer ces états, l'auteur propose de les nommer du prénom suivi d'un chiffre. Ainsi, hypnotise-t-il Lucie qui rentre dans l'état Lucie 1. Janet fait de nouveau certaines passes et Lucie 1 passe à l'état Lucie 2. Le réveil demande le passage par les mêmes étapes, mais en ordre inverse. Janet constate alors 


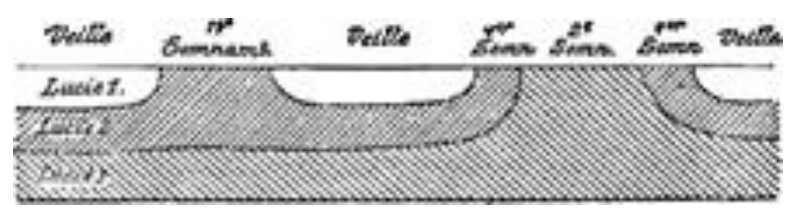

Fig. 1. Relations entre les différentes existences subconscientes et les somnambulismes successifs ([2], p. 19, ibid.).

une correspondance entre chacun de ces états, Lucie gardant le souvenir de ce qu'elle a pu faire dans l'état précédent Lucie 1. Janet propose alors le schéma suivant: Fig. 1.

Ces différentes formations (Lucie 1, Lucie 2) peuvent se développer au point de former une seconde personnalité dont les éléments ont gardé leurs associations et leur cohérence pour former une recomposition réversible. Ces deux formations peuvent alors entrer dans une forme de compétition où l'une et l'autre cherchent à s'approprier les images, les sensations et les mouvements.

Janet précise que ces deux personnalités peuvent alterner ou, au contraire, exister de façon simultanée. Dans tous les cas, la seconde personnalité (Lucie 1 par exemple) garde le souvenir de la première, ce qui n'est pas le cas pour la première (Lucie) qui ignore l'existence de celle-ci. Une autre caractéristique de cette nouvelle agrégation est qu'elle dépend d'une excitation affective dont la présence du clinicien, d'ailleurs, peut être à l'origine. Cette excitation peut être traumatique comme dans le cas de Marie où l'auteur expose trois éléments déclenchant:

- l'apparition des règles,

- la vue d'une chute mortelle,

- l'endormissement auprès d'un enfant malade (une gourme que Marie développera du côté gauche).

C'est à la question du trauma que nous sommes ici renvoyés. Parmi ces trois événements, il semble que le deuxième soit essentiellement non sexuel, c'est-à-dire psychotraumatique. Le premier et le troisième peuvent êtres associés à la question du plaisir et de la pulsion sexuelle. Le second est lui en lien direct avec la mort et apparaît comme une véritable effraction. Janet n'approfondit pourtant pas la question du sens et soigne Marie à partir de séances d'hypnose durant lesquelles il isole le souvenir traumatique pour en changer la conclusion. De fait, l'un des symptômes principaux de Marie, une image récurrente de flammes, n'est évoquée que comme une association sans signification. Apparaissent ici les limites du travail thérapeutique et l'absence de sens donné aux symptômes. Leur valeur traumatique n'a aucun lien avec les tendances de Marie qui pourtant font certainement l'objet d'un refoulement.

Janet insiste sur un autre phénomène qu'il étudie en profondeur. Il s'agit de l'exécution des suggestions posthypnotiques. Les patients dont il s'occupe réalisent des actions suggérées en état hypnotique après que l'état de veille a été retrouvé. Ces actes peuvent être assez compliqués e1 permettent par exemple de compter (des jours, Léonie se rendant à un rendez-vous au moment où il lui est demandé) ou d'écrire. Ces actes s'accomplissent soit dans un état somnambulique, qui se déclenche de lui-même et ne permet pas à la personne d'être consciente de ce qu'elle fait, soit dans un état de veille normal (ou presque normal). Janet affirme alors qu'il n'y a pas de différence fonctionnelle entre ces deux types d'exécution. Il propose alors un nouveau schéma: Fig. 2.

La dissociation est ici décrite comme un mécanisme non défensif qui existe suite à la présence, dans le cas du trauma, d'un élément parasite dont la complexité organisationnelle est plus ou 


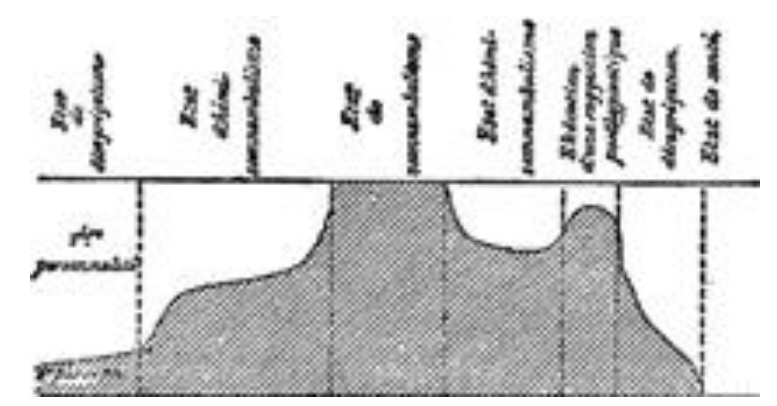

Fig. 2. Étendues relatives des diverses personnalités ([2], p. 19, ibid.).

moins grande. Avec l'hémi-somnanbulisme, nous voyons comment Janet essaie d'associer cette notion à celle d'état de conscience. S'il est difficile aujourd'hui d'accepter certaines notions comme celles de «misère psychologique » ou de «désagrégation » ou celle d'un continuum, il est pourtant intéressant de voir comment une forme d'acte, une image ou une perception reviennent de façon systématique.

Cet élément parasite, Janet le nommera «idée fixe». Malgré le terme d'idée, L. Crocq avance qu'il s'agit en fait de la souvenance de sensations, d'images, d'éprouvés qui n'a pu être associée aux représentations dans le mouvement de synthèse de la conscience. Janet, reprenant Charcot, évoque alors clairement le fait que «l'émotion causée par un accident, le «nervous shock», provoquait un état mental analogue à l'hypnotisme ou, du moins, différent de l'état psychologique normal, pendant lequel l'idée de blessure, de paralysie pénétrait dans l'esprit. La conscience revenue à l'état normal, cette idée persistait néanmoins au-dessous et arrêtait, «inhibait » tous les mouvements que le malade voulait faire » ([2], p. 106-107). Il semble donc bien qu'une idée soit apparue au moment même de l'expérience traumatique. L'émotion est alors considérée comme ayant une «action dissolvante sur l'esprit» ([2], p. 186), elle diminue la capacité de synthèse et permet à l'idée de se créer une existence autonome. Nous voyons ici que cet état mental se rapproche du vécu du sujet tel qu'il est décrit dans la dissociation péri-traumatique.

Pour expliquer qu'une idée puisse s'imposer à la conscience alors que l'association a été impossible, Janet évoque deux types de synthèse. La première, élémentaire, lie les idées entre elles alors que la seconde, actuelle, est un effort continuel répété à l'origine de l'unité du moi et de la perception personnelle. Celle-ci peut être affaiblie ou détruite alors que la seconde se maintiendra, phénomène qui sera à l'origine de l'apparition d'une idée fixe à la conscience. Janet la compare à un rêve qui se poursuit dans l'état de veille. Le schéma proposé plus haut montre que l'idée fixe apparaîtra plus aisément dans les états proches d'états somnambuliques, dits hypnoïdes.

Nous savons le sort qui sera fait à cette conceptualisation qui regroupe états hystériques et post-traumatiques. Freud affirmera que l'hypothèse des états hypnoïdes n'est d'aucune utilité pour expliquer la symptomatologie hystérique qui constitue une formation de compromis entre deux instances en conflit.

Il est intéressant de voir l'analogie pouvant exister entre la théorie actuelle du trauma et celle que Janet nous propose. Le rôle des émotions y est central.

Nous avons vu que la désagrégation était liée chez Janet à un bouleversement émotionnel qu'il considère comme venant empêcher la réalisation d'une synthèse psychique. Cette hypothèse repose sur une conception des émotions qui les opposent à la pensée consciente. Leur action est désorganisatrice et ramène le psychisme à un fonctionnement plus élémentaire propre à l'automatisme. Il reste à voir si une conception des émotions leur accordant une action spéci- 
fique peut nous aider à considérer la dissociation comme le résultat d'une tendance elle-même spécifique. C'est ce que nous allons aborder avec la théorie des émotions de N. Frijda.

\section{5. Émotion et motivation : la conception de Frijda}

Nico H. Frijda (2003) [4] parle lui de passion et d'état de motivation. Il considère la passion comme «une motivation à poursuivre un but émotionnel, soit en pensée, soit en action, avec une préséance sur d'autres préoccupations possibles ». Ces motivations sont relationnelles et cherchent à modifier la relation du sujet au monde. La tendance à l'action est donc une caractéristique intrinsèque aux émotions. Dans la peur, il existe une tendance à s'éloigner ou à se protéger. Un des aspects intéressant de la théorie de Frijda est la conceptualisation d'actions directes qui agissent sur la relation au monde par approche ou recul, ouverture ou fermeture sensorielle (comme dans la peur: recul et protection de soi pour se rendre moins vulnérable) et d'actions indirectes qui sont de nature à influencer autrui (la colère vise à empêcher autrui de nuire à nos intérêts). Dans la peur, la préparation à l'action s'oriente ainsi vers l'évitement.

L'aspect motivationnel a ceci d'intéressant qu'il expliquerait la flexibilité des réponses et rendrait compte de l'expérience d'impulsion, de l'équifinalité (c'est-à-dire de l'équivalence du but par les différentes actions), de la temporalité de l'exécution qui nécessite une explication dynamique, de la persistance de la tendance malgré les obstacles et les interruptions et de l'activation latente.

\subsection{Changement dans la préparation à l'action et expérience émotionnelle}

Si la plupart des expressions permettent de connaître l'état émotionnel du sujet, les expressions émotionnelles peuvent pourtant apparaître avec des émotions différentes comme le fait de pleurer de colère, de frustration ou de tristesse. Cette remarque, qui pourrait aller à l'encontre de l'argument précédent (relation intrinsèque entre expression et émotion), est en fait utilisée par Frijda pour distinguer un troisième élément qu'il nomme «tendance à l'action » ou plus généralement «changement dans la préparation à l'action ». Les émotions sont alors considérées comme des tendances à l'action utilisant différentes voies expressives et comportementales pour réguler la relation du sujet à l'environnement.

L'auteur propose alors d'attribuer à toute «émotion propre» une tendance à l'action, un étal final et une fonction. La peur possède ainsi une fonction de protection que la tendance à l'évitement réalise et atteint par le fait de se rendre inaccessible. La colère est accompagnée d'une tendance à l'attaque permettant un regain de contrôle et de liberté d'action. Les tendances à l'action n'ont pas à proprement parler de but à atteindre mais des «changements à achever (ou de statu quo à maintenir) tout en respectant la situation actuelle. L'émotion se distingue, par-là, de l'intention et ne peut donc s'imposer que par ce que Frijda nomme la «priorité de contrôle », c'est-à-dire le caractère nécessaire ou pulsionnel, se traduisant dans le comportement par un aspect involontaire et sur le sujet l'impression de passivité.

Frijda distingue différents niveaux affectifs où se manifeste plus ou moins l'intention, qu'il reconnaît très présente dans l'expérience émotionnelle humaine. Ceci lui permet de réaffirmer qu'il entend par émotion la forme primordiale de la vie affective que la pensée réflexive et les processus cognitifs tendent à nous faire méconnaître. En cela l'émotion est un mécanisme non réflexif dont il est pourtant possible de vivre l'expérience. Cette expérience est décrite comme:

- la conscience de la structure situationnelle significative;

- la conscience de la réaction physiologique; 
- la conscience de la préparation à l'action.

C'est-à-dire des modes directement corrélés aux sources d'information dont ils s'originent. $\mathrm{S}$ 'ajoute à ces consciences la qualité d'hédonisme (plaisant-déplaisant) propre à toute émotion et la signification ${ }^{1}$ engendrée par l'émotion elle-même.

\subsection{Processus de régulation et mécanismes de défense}

Pour achever son analyse, Frijda introduit un système de régulation qui décrit le contrôle des émotions par le sujet. Il définit la régulation comme «l'occurrence de processus dont la fonction est de modifier d'autres processus-action, expériences élicitées par une situation donnée. De telles modifications consistent en atténuation, élévation ou, dans l'événement, transformation » ([2], p. 408). Cette fonction s'attache d'une part aux conséquences du comportement et entre donc directement dans le domaine de l'inhibition et du self-control et, d'autre part, à la nature même de l'expérience émotionnelle dont la régulation provient du coping intrapsychique et du changement dans l'évaluation. Interviennent aussi des sources de régulation sociales qui tiennent compte des répercussions de certains comportements.

Nous ne pouvons revenir sur l'ensemble des mécanismes exposés, mais il est intéressant de considérer certains mécanismes de régulation considérés comme des mécanismes de défense:

- L'inhibition intervient par exemple dans le sursaut ou la réaction nommée «freeze », mais aussi dans l'anxiété qui serait une réponse à l'inhibition comportementale. Frijda y associe le déni qui apparaît lors de l'état post-traumatique [15] et qui s'accompagne de symptômes divers ; rester hébété, incapacité à apprécier la signification d'un stimulus, amnésie, perte de sens de la réalité, effroi.

- Le déni ici pris dans le sens d'une pensée d'évitement, d'omission de certaines informations ou de la gravité et de l'urgence d'une situation.

- L'effroi et la dépersonnalisation que Frijda associe aux deux premiers en faisant référence au détachement, à une évaluation défensive qui vient affaiblir ou rompre le sens de l'implication du sujet. L'effroi correspondrait à une interruption rapide du processus d'évaluation et de réception des inputs, le détachement à une interruption avant que les pensées ne soient associées à une implication personnelle, le déni à la non-génération d'implication, la non-considération des faits et à la sélection permettant une implication favorable, comme pour le refoulement (Freud), les événements pertinents pour les concerns doivent être évalués de façon défensive en permanence.

Un autre mécanisme tend à contrôler la préparation à l'action. Frijda le nomme contrôle réflexif. «La tendance à l'action est transformée en un simple «plan », une simple intention sans activation ou directe instigation à agir. Les tendances à l'action deviennent purement conceptuelles, les émotions sont transformées en «feelings»; elle peuvent ainsi être objets de réflexion » ([2], p. 442).

Frijda considère donc les mécanismes de défense comme le résultat d'une action du système de régulation sans pour autant remettre en cause sa thèse fonctionnaliste. L'intervention de la régulation lors de l'évaluation et de la préparation à l'action est jugée dans ce cas présent comme a-fonctionnelle, c'est-à-dire ayant un impact sur le fonctionnement psychique, mais relevant

\footnotetext{
${ }^{1}$ Le terme utilisé par Frijda, «significance», implique la notion de signification, mais aussi celles d'importance et de conséquence. Nous avons fait le choix de retenir le seul terme de signification.
} 
directement de la fonction de protection. Ceci est aussi vrai pour des mécanismes de faible intensité comme l'inhibition d'un comportement que pour des mécanismes beaucoup plus puissants comme le déni ou l'effroi. Ce dernier est d'ailleurs ici considéré comme un mécanisme de régulation et non comme une émotion.

En reprenant le terme de tendance, Nico Frijda s'inscrit dans une longue tradition de la théorie de l'action qui veut qu'elle soit avant tout une tendance que le dépassement d'un seuil amène à se réaliser. Cette notion est au centre de la théorie de Janet qui les considère comme des « dispositions à réagir toujours de la même manière » ([16], p. 583) chaque tendance, pour se réaliser en action, nécessite une activation dont la force diffère selon les différents degrés : la latence qui est une simple disposition, non perceptible, l'érection, le désir et l'accomplissement de l'action.

Le sentiment n'est alors pas considéré comme un activateur, mais comme un régulateur de l'activation venant la restreindre ou au contraire l'augmenter.

La théorie de N. Frijda permet de donner un fondement psychoaffectif à la dissociation. «L'évitement» dont nous avons vu qu'il en constituait la dynamique serait une tendance plus générale propre à la peur. Dans cette conception, la dissociation trouverait sa motivation mais ne pourrait être considérée comme un mécanisme de défense général. Il s'agirait d'une réaction tout à fait spécifique à un vécu émotionnel.

\section{Discussion}

\subsection{La dissociation: mécanisme ou motivation}

Nous avons vu que la dissociation était aujourd'hui considérée dans son aspect péritraumatique, c'est-à-dire apparaissant au moment même du trauma et post-traumatique, c'est-à-dire amenant à considérer qu'une partie de la personnalité du sujet semble se former en dehors du champ de la conscience. La notion de désagrégation de Janet a l'avantage de réunir ces deux phénomènes, mais repose sur une conception désorganisatrice de l'émotion. La dissociation renvoie alors à un phénomène observable, conséquence d'un dysfonctionnement psychique, plutôt qu'à un mécanisme spécifique.

Nous voyons que Frijda redonne aux émotions, en leur accordant une fonction, un sens lié à la situation dans laquelle le sujet se trouve. Le détachement qu'évoque Frijda se rapproche du vécu péri-traumatique de déréalisation et de dépersonnalisation qu'évoquent les patients. Mais plutôt que de parler de mécanisme de régulation, ne peut-on pas parler de motivation dissociative, c'est-à-dire d'une tendance à rompre l'implication du sujet dans la situation ? Nous pourrions ainsi penser qu'il existe une émotion spécifique à l'origine de cette motivation, il s'agirait alors de l'effroi.

La question reste ouverte, mais les conceptions actuelles de l'émotion peuvent nous aider à définir autrement la dissociation et à ouvrir de nouvelles pistes de recherche.

\subsection{L'étiologie traumatique}

Cette conception tend à faire du trauma un élément étiologique incontournable. Pourtant, pour Janet, le trauma apparaît davantage comme le déclencheur d'un état déjà présent chez l'individu. La difficulté de synthèse serait préexistante et se manifesterait de manière radicale dans l'expérience traumatique. Il n'y aurait aucune raison de généraliser le trauma et de chercher une thérapeutique consistant en sa découverte. Cette thèse est aujourd'hui très largement discutée puisque le trauma apparaît comme possédant son étiologie propre au-delà de toute prédisposition [17]. Il donne lieu 
à un tableau clinique délimité dont la présence d'un événement réel et le syndrome de répétition composent l'aspect principal.

L'idée fixe, pour Janet, n'est ainsi que rarement liée simplement à un événement lourd présupposé comme cause. Celui-ci peut tout aussi bien être à l'origine d'une désagrégation psychique qui permettra le développement d'une idée fixe antérieure. L'idée fixe s'enrichit ainsi de plusieurs expériences et ne peut être corrélée à un événement unique.

Or cette conception repose sur l'étude de l'hystérie. Nous avons vu que le traumatisme reposait aujourd'hui sur l'identification d'une expérience menaçant l'intégrité physique et psychique. Le cas de Marie évoqué plus haut montre que Janet considère de manière équivalente les différentes expériences pour peu qu'elles soient à l'origine d'un bouleversement émotionnel. On admettra aujourd'hui que le symptôme hystérique se distingue du symptôme traumatique et que l'étiologie soit elle aussi différente. Il apparaît ainsi que le syndrome post-traumatique soit de nature spécifique et qu'il puisse être rattaché au vécu traumatique même si d'autres facteurs interviennent, comme la prédisposition. Ceci donne au syndrome de répétition un caractère unique qu'il faut bien distinguer du symptôme hystérique.

\subsection{Le sens du symptôme : fixation et répétition}

Ce qui frappe dans les différentes théories abordant la question du trauma est l'importance que revêt la question de l'interprétation et du sens de la névrose. Nous constatons, en effet, que le souvenir traumatique semble tout à fait dénué de sens et de valeur symbolique. Les auteurs évoquent d'ailleurs une absence de possibilité d'associer l'image traumatique au réseau de représentations psychiques. Apparaît alors la nécessité de faire intervenir un élément extérieur dont la nature même est d'être dénuée de toute signification. Janet fait ainsi appel à l'automatisme qu'il définit comme une fonction innée du psychisme régissant les contenus élémentaires de la psyché. Cette action se suffit à elle-même et contrecarre l'activité de synthèse propre aux couches supérieures de la pensée.

Il en est de même chez Freud où une compulsion va venir régir la répétition (Freud, 1920) ${ }^{2}$. En faisant intervenir une tendance innée à tout être humain, Freud considère la répétition comme se suffisant à elle-même, résolument hors du champ de la signification, c'est-à-dire ne se rattachant à rien qui en expliquerait le dynamisme. Il ne s'agit plus de dualisme, mais d'une tendance absolue à la décharge. Même si la répétition du rêve traumatique est interprétée comme une recherche de maîtrise et d'abréaction de tensions excessives, il n'en reste pas moins que l'élément ultime est la pulsion de mort.

La nature de l'expérience traumatique semble ainsi se constituer d'une positivité non signifiante, d'un symptôme dont la caractéristique est d'être le résultat de l'expression d'une tendance originaire du psychisme. Même dans le cas de la théorie lacanienne, il apparaît une référence au réel dont nous savons qu'il échappe à l'ordre symbolique.

Chez Janet, nous constatons que la répétition est liée à la fixation. Il semble, en effet, que l'apparition de l'idée fixe se fasse lorsque l'individu entre dans des états de consciences différents. Les sujets se plaignent de l'intrusion des souvenirs traumatiques qui apparaitraient alors lorsque le sujet change d'état: endormissement, sommeil, état hypnotique. La répétition serait ainsi le pendant de la fixation, sa manifestation se ferait au rythme des changements des états de conscience.

\footnotetext{
${ }^{2}$ Freud S. Au-delà du principe de plaisir. In: Essais de psychanalyse (1920), [18].
} 


\subsection{Aspects cliniques}

La question du rôle de la dissociation est aujourd'hui peu étudiée en France malgré les travaux de Janet. Il semble pourtant que la présence d'un tel vécu lors de l'expérience traumatique soit en partie prédictive de l'apparition de la symptomatologie. Une meilleure compréhension de ce phénomène peut donc nous aider à affiner le travail clinique avec les patients. Nous remarquons, par exemple, que nombre d'entre eux hésitent à parler d'un vécu dissociatif par crainte d'y voir un moment de folie, parce qu'ils pensent que cela n'a pas d'importance ou tout simplement que l'évoquer peut le redéclencher. Il apparaît pourtant qu'un tel phénomène ne soit pas seulement péri-traumatique, mais bien central et qu'il se maintient après le trauma. En le liant à l'effroi, nous pouvons reconnaître la spécificité d'un tel vécu émotionnel et dégager l'émotion de son caractère désorganisant, désagrégeant que lui avait octroyé Janet. La clinique a ainsi tout intérêt à distinguer ce qui est de l'ordre de la peur, de l'angoisse et de l'effroi. En associant la motivation dissociative à l'effroi, nous espérons aider le clinicien à écouter les vécus émotionnels dans le discours du patient et à voir en eux un ensemble de motivations possédant une signification (au sens de Frijda).

Le second aspect concerne la question de la répétition. Celle-ci est souvent utilisée afin de rendre compte de la répétition des symptômes comme les cauchemars ou les flash-backs. Or c'est négliger le fait que les patients se trouvent en état de fixation, de reviviscence permanente de l'expérience traumatique. L'effet de répétition est dû à l'observation et aux changements d'état de conscience. Lorsque le patient est en face de nous, il est essentiel de l'entendre de cette place où il est maintenu en permanence, celle du moment traumatique. Les symptômes ne sont que la manifestation répétée de cette fixation, l'essentiel étant alors de permettre au patient de sortir de cette situation. L'instant de la rencontre clinique devient alors celui d'une possible reviviscence.

\section{Conclusion}

Nous avons vu que les conceptions actuelles du trauma associaient une symptomatologie au vécu d'un événement possédant des caractéristiques précises. La symptomatologie se caractérise par un syndrome de répétition considéré comme pathognomonique. Il consiste en une reviviscence de l'expérience traumatique sous la forme de flash-backs, cauchemars, vécus «as if »se manifestant de manière intrusive dans le psychisme du sujet.

Les recherches actuelles ont montré qu'il existait dans l'expérience du sujet un vécu dissociatif constitué d'une dépersonnalisation ou d'une déréalisation dont la présence au moment du trauma pouvait être prédictive de la gravité du trauma. Cette dissociation péri-traumatique est rapprochée de la dissociation telle que l'a définie Janet et qui consiste en une désagrégation psychique. Celleci consiste en une réduction du champ de la conscience et une difficulté de synthèse due à un bouleversement émotionnel.

Nous avons essayé de montrer que les travaux de Frijda autour de la fonction des émotions et de leur considération en termes de motivation pouvaient nous aider à donner à la dissociation un sens spécifique. Celle-ci ne serait alors plus liée à une désagrégation, mais se rapprocherait d'un évitement psychique radical. Cet évitement serait le propre d'une émotion spécifique, l'effroi, dont la tendance serait une motivation dissociative.

Cette hypothèse repose sur la distinction du trauma psychique d'un autre trauma tel qu'i] apparaîtrait dans l'hystérie et que Janet considérait sans distinction. Le trauma aurait alors pour conséquence de fixer les images et les sensations associées au vécu. La répétition se manifesterai1 par la réapparition de cette fixation lorsque l'état de conscience de l'individu se modifie. 
Nous voyons comment la notion de dissociation chez Janet nous permet, par sa complexité, de considérer sous un jour nouveau la question du trauma et de ses manifestations. Elle implique que soient étudiées en toute rigueur les manifestations psychopathologiques au-delà des a priori qui nous amènent trop souvent à créer un mécanisme ad hoc pour expliquer un phénomène.

La référence aux travaux de Janet dans le cadre des recherches actuelles ouvre de nouvelles perspectives de recherche autour d'une définition du trauma, de la place de la dissociation péri-traumatique et de la dissociation post-traumatique, des émotions et de la répétition.

\section{Références}

[1] Frijda NH. The emotions. Cambridge: Cambridge University Press; 1986.

[2] Janet P. L'automatisme psychologique. Essai de psychologie expérimentale sur les formes inférieures de l'activité humaine (1889). Paris: Édition Société Pierre Janet et CNRS; 1973.

[3] Bühler KE, Heim G. Introduction générale à la psychopathologie et à la psychothérapie de Pierre Janet. Ann Med Psychol 2001;159:261-72.

[4] Frijda NH. Passions : l'émotion comme motivation, dans Les émotions. In: Colletta JM, Tcherkassof A, editors. Les émotions : cognition, langage et développement. Sprimont: Mardaga; 2003. p. 15-32.

[5] Crocq L. Les traumatismes psychiques de guerre. Paris: Odile Jacob; 1999.

[6] American Psychiatric association. DSM-IV, Manuel diagnostique et statistique des troubles mentaux. Paris: Masson; 1996.

[7] Briole G, Lebigot F, Lafont B, Favre JD, Vallet D. Le traumatisme psychique : rencontre et devenir. Paris: Masson, coll. «Congrès de psychiatrie et de neurologie de langue française »; 1994.

[8] De Clerq M, Lebigot F. Les traumatismes psychiques. Paris: Masson; 2001.

[9] Thoret Y, Giraud AC, Ducerf B. La dissociation hystérique dans les textes de Janet et Freud avant 1911. Evol Psychiatr 1999;64:749-64.

[10] Bottéro A. Une histoire de la dissociation schizophrénique. Evol Psychiatr 2001;66:43-60.

[11] Michelson L, June K, Vives A, Testa S, Marchione N. The role of trauma and dissociation in cognitive-behavioral psychotherapy outcome and maintenance for panic disorder with agoraphobia. Behav Res Ther 1988;36(11):1011-50.

[12] Herman JL. Complex PTSD: Asyndrome in survivors of prolonged and repeated truma. J Traum Stress 1992:5:377-91.

[13] Shilony E, Fleming MZ. Dissociative disorders. In: Encyclopedia of human behavior. San Diego: Academic Press; 1994. p. 143-151.

[14] Gershuny BS, Thayer FJ. Relations among psychological trauma, dissociative phenomena, and trauma-related distress: a review and integration. Clin Psychol Rev 1999;19(5):631-57.

[15] Horowitz MJ. Stress response syndromes. 2nd ed. Northvale, NJ: Jason Aronson; 1986.

[16] Heim G, Bühler KE. Les idées fixes et la psychologie de l'action de Pierre Janet. Ann Med Psychol 2003;161:579-86.

[17] Gershuny BS, Cloitre M, Otto MW. Peritraumatic dissociation and PTSD severity: do eventrelated fears about death and control mediate their relation? Behav Res Ther 2003;41:157-66.

[18] Freud S. Essais de psychanalyse (1920). Petite Bibliothèque Payot; 1981. 